\title{
Epidemiological surveillance of common respiratory viruses in patients with suspected COVID-19 in Southwest China
}

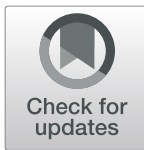

Yanjun $\mathrm{Si}^{1 \dagger}$, Zhenzhen Zhao ${ }^{1 \dagger}$, Rong Chen ${ }^{2 \dagger}$, Huiyu Zhong ${ }^{1}$, Tangyuheng Liu', Minjin Wang ${ }^{1}$, Xingbo Song ${ }^{1}$, Weimin $\mathrm{Li}^{3^{*}}$ and Binwu Ying ${ }^{1 *}$

\begin{abstract}
Background: The outbreak of coronavirus disease 2019 (COVID-19) caused by the severe acute respiratory syndrome coronavirus 2 (SARS-CoV-2) is currently the peak season of common respiratory viral infections. However, the clinical symptoms of most SARS-CoV-2 infected patients are not significantly different from those of common respiratory viral infections. Therefore, knowing the epidemiological patterns of common respiratory viruses may be valuable to improve the diagnostic and therapeutic efficacy of patients with suspected COVID-19, especially in Southwest China (a mild epidemic area).
\end{abstract}

Methods: A total of 2188 patients with clinically suspected of COVID-19 in Southwest China were recruited from January 21 to February 29, 2020. Nasopharyngeal swabs, throat swabs and sputum specimens were collected to detect SARS-CoV-2 by using real-time reverse transcription-polymerase chain reaction (RT-PCR) and other 12 viruses via PCR fragment analysis combined with capillary electrophoresis. Clinical characteristics and laboratory test findings were acquired from electronic medical records. All data were analyzed to unravel the epidemiological patterns.

Results: Only 1.1\% (24/2188) patients with suspected COVID-19 were eventually confirmed to have SARS-CoV-2 infection, and the most frequently observed symptoms were fever $(75.0 \%, 18 / 24)$ and cough $(20.8 \%, 5 / 24)$. The overall detection rate of other respiratory pathogens was 10.3\% (226/2188). Among them, human rhinovirus (3.2\%, 71/2188), human parainfluenza viruses (1.6\%,35/2188), influenza B virus (1.2\%, 26/2188) and mycoplasma pneumonia (1.2\%, 26/ 2188) were the predominantly detected pathogens in this study. Moreover, the co-infection was observed in 22 specimens. Notably, one COVID-19 case had a coexisting infection with human parainfluenza virus $(4.2 \%, 1 / 24)$ and bocavirus was the most common virus tending to occur in co-infection with other respiratory pathogens.

(Continued on next page)

\footnotetext{
*Correspondence: weimin003@163.com; yingbinwu@scu.edu.cn

${ }^{\dagger}$ Yanjun Si, Zhenzhen Zhao and Rong Chen contributed equally to this work. ${ }^{3}$ Department of Respiratory and Critical Care Medicine, West China Hospital, Sichuan University, 37 Guoxue Alley in the wuhou District, Chengdu, Sichuan, China

${ }^{1}$ Department of Laboratory Medicine, West China Hospital, Sichuan University, 37 Guoxue Alley in the wuhou District, Chengdu, Sichuan, China Full list of author information is available at the end of the article
}

(c) The Author(s). 2020 Open Access This article is licensed under a Creative Commons Attribution 4.0 International License, which permits use, sharing, adaptation, distribution and reproduction in any medium or format, as long as you give appropriate credit to the original author(s) and the source, provide a link to the Creative Commons licence, and indicate if changes were made. The images or other third party material in this article are included in the article's Creative Commons licence, unless indicated otherwise in a credit line to the material. If material is not included in the article's Creative Commons licence and your intended use is not permitted by statutory regulation or exceeds the permitted use, you will need to obtain permission directly from the copyright holder. To view a copy of this licence, visit http://creativecommons.org/licenses/by/4.0/ The Creative Commons Public Domain Dedication waiver (http://creativecommons.org/publicdomain/zero/1.0/) applies to the data made available in this article, unless otherwise stated in a credit line to the data. 


\begin{abstract}
(Continued from previous page)
Conclusions: This study reveals the epidemiological features of common respiratory viruses and their clinical impact

during the ongoing outbreak of COVID-19 in a mild epidemic area. The findings highlight the importance of

understanding the transmission patterns of the common respiratory virus in COVID-19 regions, which can provide

information support for the development of appropriate treatment plans and health policies, while eliminating

unnecessary fear and tension.
\end{abstract}

Keywords: COVID-19, Respiratory viral pathogens, Surveillance research

\section{Background}

Since its outbreak in Wuhan, China in December 2019, the severe acute respiratory syndrome coronavirus 2 (SARS-CoV-2)-infected disease (COVID-19) has spread throughout China and continuously to many other countries and regions around the world [1, 2], thus becoming a major public health threat and economic burden worldwide. The research on the clinical characteristics of COVID-19 in China has shown that the most common clinical symptoms of COVID-19 are fever (87.9\%) and cough $(67.7 \%)$, followed by fatigue (38.1\%), sputum production (33.4\%), shortness of breath (18.6\%), muscle pain or joint pain $(14.8 \%)$, sore throat $(13.9 \%)$, headache (13.6\%), chills (11.8\%) and other atypical symptoms. However, these clinical symptoms at the onset of COVID-19 are not different from common respiratory viral infections [3]. Indeed, the patients with acute respiratory infections (ARIs) caused by common respiratory viruses often manifest fever, cough, rhinitis, sore throat, headache and imaging features of pneumonia, which are similar to those of COVID-19 patients, thus leading to seasonal epidemics with pneumonia, bronchitis, severe respiratory failure, and even death $[4,5]$. These similar symptoms of ARIs and COVID-19 can hamper the diagnostic and therapeutic efficacy of patients with suspected COVID-19, particularly during the epidemic period of SARS-CoV-2. Therefore, identification of the causative respiratory pathogens is of great importance for stopping the epidemic spread of COVID-19 and contributing to the reduced duration of patient isolation, especially for those who are infected with other common respiratory viruses.

At present, the epidemic situation of COVID-19 is varied across regions. As of February 29, 2020, the National Health Commission has reported a total of 79,824 confirmed COVID-19 cases and 2870 death in Mainland China [6]. There are 143 confirmed cases found in Chengdu city, Sichuan province, southwest of China, and three of them have died [7]. Apparently, this number is much lower than that reported in Wuhan [6]. In the meantime, ARIs are the major causes of outpatient visits and hospitalization in all age groups, especially during winter and spring [8]. A surveillance study on the epidemiology of ARIs among hospitalized young children in Chengdu from 2009 to 2014 reported that 23.0\% of ARI patients were identified with human rhinovirus (HRV), 22.7\% patients with respiratory syncytial virus (RSV), $13.4 \%$ patients with human parainfluenza virus (HPIV) and 8.4\% patients with human bocavirus (HBoV) [9]. Furthermore, a systematic review of the causes of ARIs demonstrated that RSV was the most frequently detected pathogen among patients of different age groups [10]. Knowing the epidemiological patterns of common respiratory viral infections at the population scale may help in guiding optimal diagnosis and treatment strategy for ARI patients, and it is more important for areas with relatively lower rates of COVID-19, including Chengdu city. However, most epidemiological studies conducted to date have rarely focused on the distribution patterns of respiratory viral infections and their clinical impact during the ongoing outbreak of COVID19. A deeper understanding of the actual situation of respiratory viral infections in this location can provide information support for the development of appropriate treatment plans and health policies, while eliminating unnecessary fear and tension. Therefore, in this study, we aimed to investigate the epidemiological, clinical and virologic characteristics of patients with suspected COVID-19, and clarify the actual infection rate and epidemic situation of SARS-CoV-2 and other respiratory pathogens outside the main epidemic area of COVID19, particularly in Southwest China.

\section{Method \\ Study design and patients}

This was a retrospective research investigated on the epidemic situation of SARS-CoV-2 and other viruses in the West China Hospital of Sichuan University, Chengdu, China (a region with mild morbidity of COVID-19), from 21 January to 29 February 2020. The eligibility criteria for the patients enrolled in this study were as follows: (i) fever $\left(\geq 37.0^{\circ} \mathrm{C}\right)$ and/or respiratory symptoms (e.g., cough, sputum production, hemoptysis, shortness of breath, wheezing, and chest pain, etc.); (ii) with no other underlying pulmonary diseases; and (iii) underwent screening test for 13 respiratory viral pathogens, including SARS-CoV-2. The detailed information, such as clinical manifestations, diagnosis and laboratory test findings, were recorded in case report forms. 
Patients' demographics and baseline characteristics were also collected. This study was approved by the Biomedical Research Ethics Committee of West China Hospital (ChiCTR2000030542), and written informed consent was obtained from all participants or their parents/caregivers before sample collection.

\section{Viral RNA isolation and sample processing}

Nasopharyngeal swabs, throat swabs and sputum specimens were collected from patients with clinically suspected COVID-19. After sampling, the specimens were placed into collection tubes filled with a $2 \mathrm{~mL}$ virus preservation solution. Total viral RNA was extracted using a QIAamp viral RNA mini kit (Qiagen, Germany). Briefly, $200 \mu \mathrm{L}$ of each specimen was transferred into a tube containing $210 \mu \mathrm{L}$ lysis solution and $20 \mu \mathrm{L}$ proteinase $\mathrm{K}$, followed by incubation at $56^{\circ} \mathrm{C}$ for 30 min with vigorous shaking. The obtained cell lysates were transferred to a collection tube and then added with a washing buffer. After centrifugation, the bound RNA extracts were eluted to a final volume of $50 \mu \mathrm{L}$.

\section{SARS-CoV-2 real-time quantitative PCR}

Real-time reverse transcription-polymerase chain reaction (RT-PCR) was performed by amplifying two target genes, including open reading frame $1 \mathrm{ab}$ (ORF1ab) and nucleocapsid protein $(\mathrm{N})$.

Target 1 (ORF1ab):

Forward primer: CCCTGTGGGTTTTACACTTAA, Reverse primer: ACGATTGTGCATCAGCTGA,

Fluorescent probe (P): 5'-FAM-CCGTCTGCGGTATG TGGAAAGGTTATGG-BHQ1-3';

Target two $(\mathrm{N})$ :

Forward primer: GGGGAACTTCTCCTGCTAGAAT, Reverse primer: CAGACATTTTGCTCTCAAGCTG,

Fluorescent probe (P): 5 '-FAM-TTGCTGCTGCTTGA CAGATT-TAMRA-3'.

Real-time RT-PCR assays were performed on an ABI 7500 system (Applied Biosystems instruments, USA) using a RT-PCR kit (Sansure Biotech Inc., China). Each $30 \mu \mathrm{L}$ reaction mixture contained $18.5 \mu \mathrm{L}$ of one-step RT-PCR reaction buffer, $1.5 \mu \mathrm{L}$ of enzyme mix and $10 \mu \mathrm{L}$ of RNA as a template. Thermal cycling was initiated at $50{ }^{\circ} \mathrm{C}$ for $20 \mathrm{~min}$ (for reverse transcription), followed by $95^{\circ} \mathrm{C}$ for $10 \mathrm{~min}$ and 40 cycles of $95^{\circ} \mathrm{C}$ for $15 \mathrm{~s}$ and $60^{\circ} \mathrm{C}$ for $30 \mathrm{~s}$ using the CFX96 Touch RT-PCR system (BIO-RAD Laboratories).

\section{The multiplex platform for respiratory pathogen panel testing}

The nucleic acids of each sample were subjected to multiplex amplification using a Respiratory Pathogen Multiplex Detection Kit (Ningbo Health Gene Technology, Ningbo, China) on an ABI Verity 96 Thermal
Cycler (Thermo Fisher Scientific, Carlsbad, CA, USA). The PCR product was then subjected to capillary electrophoresis and fragment analysis using a 3500Dx Genetic Analyzer (Thermo Fisher Scientific, Carlsbad, CA, USA) according to the manufacturer's protocol. The panel was specifically designed to detect 12 pathogens, namely, influenza A (H1N1) 2009 virus (09H1N1), seasonal $\mathrm{H} 3 \mathrm{~N} 2$ virus (H3N2), influenza B virus (InfB), human adenovirus (HADV), HBoV, human coronavirus (HCOV; 229E, OC43, NL63, HKU1), human metapneumovirus (HMPV), HPIV, HRV, human respiratory syncytial virus (HRSV), mycoplasma pneumonia (Mp) and chlamydia $(\mathrm{Ch})$, within $4 \mathrm{~h}$.

\section{Statistical analyses}

Kolmogorov-Smirnov test was used to assess the normality of the measurement data. The continuous variables with normal distribution were presented as mean \pm standard deviation (SD), while the non-normally distributed variables were expressed as median (interquartile range, IQR). Pearson's chi-square test, MannWhitney U test and Student's t-test were used whenever appropriate. The categorical variables were expressed as numbers and percentages. Statistical analysis was performed using SPSS 22.0 and a two-sided $p$-value of $\leq 0.05$ was regarded as statistically significant in this study.

\section{Results \\ Clinical and epidemiological features of COVID-19 patients}

In total, 2188 individuals presenting with suspicious symptoms of COVID-19 were included. Twenty-four patients were diagnosed with COVID-19, with a positive rate of $1.1 \%$. Of these patients, the average age was 46.2 years and $54.2 \%(13 / 24)$ were men. Fever $(75.0 \%, 18 / 24)$ was the most frequent symptom, followed by cough $(20.8 \%, 5 / 24)$. Abnormal findings were observed on the chest computed tomography scans in most patients (91.7\%, 22/24). Besides, one COVID-19 case had coexisting HPIV infection $(4.2 \%, 1 / 24)$, and the remaining COVID-19 patients were free from the other 12 respiratory viral pathogens.

\section{Profiles of other respiratory viruses among cases with suspected COVID-19}

There were 226 cases out of 2188 suspected COVID-19 patients were confirmed as other respiratory viruses positive. Among them, 47.8\% (108/226) were male, with an average age of 41.3 years. The overall detection rate was $10.3 \%(226 / 2188)$. As shown in Table 1 , the most frequently detected pathogens included $\operatorname{HRV}(3.2 \%, 71 /$ 2188), HMPV (1.6\%, 35/2188) and $\operatorname{InfB}(1.2 \%, 26 / 2188)$. Moreover, 38 patients were positive for the influenza 
Table 1 The characteristics of respiratory viral pathogens from suspicious COVID-19 patients

\begin{tabular}{|c|c|c|c|c|c|c|}
\hline Viral pathogen & All positive & Mono virus & Two viruses & Triple or more viruses & Detection rate & Virus specific co-infection proportion \\
\hline SARS-CoV-2 & 24 & 23 & 1 & - & $1.1 \%$ & $4.2 \%$ \\
\hline HRV & 71 & 56 & 14 & 1 & $3.2 \%$ & $21.1 \%$ \\
\hline HMPV & 35 & 34 & 1 & - & $1.6 \%$ & $2.9 \%$ \\
\hline $\mathrm{Mp}$ & 26 & 25 & - & 1 & $1.2 \%$ & $3.8 \%$ \\
\hline $\operatorname{lnfB}$ & 26 & 24 & 2 & - & $1.2 \%$ & $7.7 \%$ \\
\hline HRSV & 17 & 15 & 2 & - & $0.8 \%$ & $11.8 \%$ \\
\hline HADV & 14 & 12 & 2 & - & $0.6 \%$ & $14.3 \%$ \\
\hline $\mathrm{HBOV}$ & 14 & 2 & 11 & 1 & $0.6 \%$ & $85.7 \%$ \\
\hline HPIV & 13 & 11 & 2 & - & $0.6 \%$ & $15.4 \%$ \\
\hline $\mathrm{HCOV}$ & 11 & 6 & 5 & - & $0.5 \%$ & $45.5 \%$ \\
\hline $\mathrm{Ch}$ & 9 & 9 & - & - & $0.4 \%$ & - \\
\hline H3N2 & 7 & 6 & 1 & - & $0.3 \%$ & $14.3 \%$ \\
\hline 09H1N1 & 5 & 5 & - & - & $0.2 \%$ & - \\
\hline
\end{tabular}

Abbreviations: SARS-CoV-2 severe acute respiratory syndrome coronavirus 2, HRV human rhinovirus, HMPV human metapneumovirus, Mp mycoplasma pneumoniae, InfB influenza B virus, HRSV human respiratory syncytial virus, HADV human adenovirus, HBoV human bocavirus, HPIV human parainfluenza viruses, HCOV human coronavirus (229E, OC43, NL63, HKU1), Ch chlamydia. H3N2, seasonal H3N2 virus; 09H1N1, influenza A virus H1N1 (2009)

virus, of which $68.4 \%(26 / 38)$ were co-existed with InfB infection. Twelve patients infected with InfA were classified as the subgroups $09 \mathrm{H} 1 \mathrm{~N} 1(41.7 \%, 5 / 12)$ and $\mathrm{H} 3 \mathrm{~N} 2$ $(58.3 \%, 7 / 12)$. Of the remaining viral pathogens detected, the positive detection rates were $1.2 \%(26 / 2188)$ for $\mathrm{Mp}$, $0.8 \%(17 / 2188)$ for HRSV, $0.6 \%$ (14/2188) for both HADV and HBoV, $0.6 \%$ (13/2188) for HPIV, $0.5 \%$ for HCOV $(11 / 2188)$, and the rarest pathogen detected was Ch $(0.4 \%, 9 / 2188)$. Besides, the overall detection rate of respiratory virus co-infection was $1.0 \%$ (22/2188).

Eleven patterns of co-infection (simultaneous infection with two or more viruses) were evaluated in this surveillance research. The detailed information on these coinfection patterns is summarized in Table 2. Of the 226 cases infected with other respiratory viruses, there were $22(9.7 \%)$ cases co-infected with other respiratory pathogens. The co-infection patterns of two or more viruses are provided in Table 2. The most dominant coinfectious agents were $\mathrm{HBoV}$ and $\mathrm{HRV}$, accounting for $45.5 \%(10 / 22)$ of all co-infection cases. Moreover, it was found that $85.7 \%(12 / 14)$ of $\mathrm{HBoV}$-positive cases had coinfections with other viruses.

\section{Comparison of laboratory features between patients with COVID-19 and other respiratory infections}

As shown in Table 3, the values of most laboratory indicators were relatively similar between patients with COVID-19 patients and those infected with other respiratory pathogens. There were no significant discrepancies between the two groups concerning age and gender distributions. The levels of triglyceride (TG) and alkaline phosphatase (ALP) were increased and decreased in COVID-19 cases, respectively, when compared to those infected with other respiratory pathogens $(p<0.05)$.

\section{Discussion}

Since the first case of COVID-19 was identified in Wuhan, China, in late December 2019, the outbreak has gradually spread nationwide, and is now rapidly spreading across many countries worldwide. In the meantime, it is also the peak season for respiratory tract infections, and their severe clinical symptoms and cross-species transmission patterns pose a huge threat to human health. Therefore, it is vital for clinicians to timely and

Table 2 Co-infections types of double and triple viruses

\begin{tabular}{llll}
\hline Number & Type & co-infectious pathogens & Cases \\
\hline 1 & HBOV +HRV & 2 & 10 \\
2 & HCOV+HRV & 2 & 2 \\
3 & HCOV+HMPV & 2 & 1 \\
4 & HCOV+HPIV & 2 & 1 \\
5 & HCOV+InfB & 2 & 1 \\
6 & H3N2 + HRSV & 2 & 1 \\
7 & HADV+HRV & 2 & 1 \\
8 & HADV+ HBoV & 2 & 1 \\
9 & HRSV+HPIV & 2 & 1 \\
10 & HRV + InfB & 2 & 1 \\
11 & SARS-CoV-2 + HPIV & 2 & 1 \\
12 & HBoV +HRV + Mp & 3 & 1 \\
\hline
\end{tabular}

Abbreviations: HBoV human bocavirus, HRV human rhinovirus, HCOV human coronavirus (229E, OC43, NL63, HKU1), H3N2 influenza A seasonal H3N2 virus, HRSV human respiratory syncytial virus, HADV human adenovirus, HPIV human parainfluenza viruses, InfB influenza $B$ virus, HMPV human metapneumovirus, Mp mycoplasma pneumoniae 
Table 3 Laboratory features of the COVID-19 patients and patients with other respiratory virus infections

\begin{tabular}{|c|c|c|c|}
\hline Characteristics & Cases with COVID-19 & Cases with other viral infections & $P$ \\
\hline Age - years & $46.2 \pm 17.4$ & $41.3 \pm 19.3$ & 0.238 \\
\hline Gender-M(\%) & $13(54.2)$ & $108(47.8)$ & 0.552 \\
\hline Erythrocyte $\left(\times 10^{12} / \mathrm{L}\right)$ & $4.7 \pm 0.6$ & $4.5 \pm 0.9$ & 0.505 \\
\hline Hemoglobin (g/L) & $142.3 \pm 18.0$ & $135.2 \pm 26.9$ & 0.376 \\
\hline Hematocritc & $0.4 \pm 0.1$ & $0.4 \pm 0.1$ & 0.569 \\
\hline Platelets $\left(\times 10^{9} / \mathrm{L}\right)$ & $204.3 \pm 72.0$ & $199.3 \pm 70.0$ & 0.789 \\
\hline Leucocytes $\left(\times 10^{9} / \mathrm{L}\right)$ & $5.3(3.7-10.2)$ & $7.2(5.6-9.8)$ & 0.095 \\
\hline Neutrophils (\%) & $67.8 \pm 13.9$ & $68.3 \pm 14.1$ & 0.893 \\
\hline Lymphocytes (\%) & $22.4 \pm 11.2$ & $20.1 \pm 11.3$ & 0.438 \\
\hline Monocytes (\%) & $8.9 \pm 3.3$ & $8.3 \pm 3.4$ & 0.559 \\
\hline TBIL (umol/L) & $8.6(6.2-20.0)$ & $8.8(6.7-12.5)$ & 0.762 \\
\hline DBIL (umol/L) & $3.3(2.1-6.0)$ & $3.2(2.2-5.0)$ & 0.886 \\
\hline $\mathrm{ALT}(\mathrm{IU} / \mathrm{L})$ & $18.5(12.0-22.8)$ & $17.0(12.0-30.0)$ & 0.988 \\
\hline AST (IU/L) & $21.0(14.0-27.8)$ & $22.0(17.0-29.0)$ & 0.399 \\
\hline $\mathrm{TP}(\mathrm{g} / \mathrm{L})$ & $69.9 \pm 5.1$ & $71.8 \pm 10.0$ & 0.515 \\
\hline $\mathrm{ALB}(\mathrm{g} / \mathrm{L})$ & $42.3 \pm 3.6$ & $41.5 \pm 7.8$ & 0.751 \\
\hline $\mathrm{GLB}(\mathrm{g} / \mathrm{L})$ & $27.7 \pm 3.7$ & $30.3 \pm 5.9$ & 0.134 \\
\hline$A / G$ & $1.6 \pm 0.3$ & $1.4 \pm 0.4$ & 0.247 \\
\hline GLU (mmol/L) & $6.2 \pm 1.5$ & $6.7 \pm 2.2$ & 0.463 \\
\hline UREA (mmol/L) & $4.6(3.0-5.5)$ & $4.1(3.3-6.2)$ & 0.865 \\
\hline CREA (umol/L) & $82.5(60.5-97.8)$ & $72.5(61.3-88.5)$ & 0.579 \\
\hline Cys-C (mg/L) & $0.9 \pm 0.3$ & $1.1 \pm 0.8$ & 0.481 \\
\hline URIC (umol/L) & $291.5 \pm 112.5$ & $294.5 \pm 95.3$ & 0.921 \\
\hline $\mathrm{TG}(\mathrm{mmol} / \mathrm{L})$ & $2.2 \pm 1.5$ & $1.3 \pm 0.8$ & 0.003 \\
\hline $\mathrm{CHOL}(\mathrm{mmol} / \mathrm{L})$ & $3.8 \pm 0.9$ & $3.9 \pm 1.1$ & 0.762 \\
\hline $\mathrm{HDL}-\mathrm{C}(\mathrm{mmol} / \mathrm{L})$ & $1.1 \pm 0.3$ & $1.1 \pm 0.5$ & 0.740 \\
\hline $\mathrm{LDL}-\mathrm{C}(\mathrm{mmol} / \mathrm{L})$ & $2.3 \pm 0.6$ & $2.3 \pm 0.9$ & 0.426 \\
\hline ALP (IU/L) & $61.5(53.5-68.8)$ & $82.0(65.0-97.0)$ & 0.002 \\
\hline GGT (IU/L) & $21.0(11.8-34.0)$ & $23.0(16.0-46.0)$ & 0.267 \\
\hline CK (IU/L) & $107.0(74.8-118.0)$ & $77.0(51.0-116.0)$ & 0.261 \\
\hline LDH (IU/L) & $180.5(154.8-212.5)$ & $184.0(166.3-238.5)$ & 0.251 \\
\hline $\mathrm{HBDH}(\mathrm{IU} / \mathrm{L})$ & 139.5 (118.3-170.0) & $139.0(122.5-181.0)$ & 0.607 \\
\hline TBA (umol/L) & $2.8(1.9-5.6)$ & $5.0(3.32-7.15)$ & 0.088 \\
\hline
\end{tabular}

accurately identify patients who are very likely to have COVID-19. However, it is difficult to distinguish between the patients with ARIs and COVID-19, mainly since their clinical manifestations are nearly identical at the outset. Although the etiology of ARIs is complicated, some common respiratory viruses have been reported as the leading causes of the disease [11, 12]. Hence, we should attach great importance to ARI patients infected with other viral pathogens, and at the same time, clarify the actual infection rate of SARS-CoV-2 in Southwest China. This may help us to better control the epidemic outbreak and correctly handle other respiratory tract infections.

In this study, a total of 24 patients were confirmed to have COVID-19 infection, with a positive detection rate of 1.1\%. Up until 29 February 2020, 538 COVID-19 cases had been diagnosed in Sichuan province, and 143 of them were from Chengdu. However, a total of 67,773 
patients were infected with COVID-19 in Hubei. Sichuan province ranked 11th in the country regarding the number of COVID-19 cases and is thus considered a mild epidemic area. The West China Hospital of Sichuan University belongs to a comprehensive tertiary hospital rather than a communicable disease hospital or designated hospital during the early outbreak period. Besides, $10.3 \%$ of the suspected COVID-19 patients were positive for other respiratory viruses. Moreover, the infection rates of common respiratory viruses were much higher compared to the SARS-CoV-2 infection. These results indicate that we should simultaneously pay close attention to ARIs patients caused by other respiratory viral pathogens, particularly in a mild epidemic area, even during the COVID-19 outbreak.

Among the 12 types of common respiratory pathogens, the most are HRV (3.2\%, 71/2188), which also represents one of the most important etiological agents for respiratory tract infection. One study conducted by Kong et al. [13] showed that the positive rate of rhinovirus/enterovirus was $4.8 \%$ among 806 adult patients with ARIs in Shanghai. HMPV is a leading cause of ARIs, which exhibits a positive rate of $1.6 \%$ in our cases with suspected COVID-19. Consistently, Li et al. [14]. have reported a positive rate of 1.7\% for HMPV in 2936 patients with ARIs in Beijing, China. It is worth noting that most studies have focused on the epidemiological features of HMPV in the vulnerable population. For instance, Zhang and colleagues [15] performed a cross-sectional study in Guangzhou, and reported that 103 out of 5133 pediatric patients with ARIs (2.0\%) were positive for HMPV. In the present study, 38 patients were positive for influenza virus infection, of which $68.4 \%$ were InfB infection, and the remaining were InfA infection. These results indicate that InfB is the most predominant respiratory pathogen, which may differ from those reported in other studies $[13,16]$. The findings of Kong et al. [13] showed that the positive rates of InfA and InfB were 40.5 and $14.4 \%$, respectively. Another study conducted in Chongqing demonstrated a positive rate of $13.3 \%$ for influenza viruses among 24,868 patients with influenza-like illnesses (65.7\% InfA, 34.1\% InfB, and 0.2\% co-infection of InfA and InfB co-infection) [16]. The geographical location difference, limited sample size and research duration may partly explain these contradictory findings, particularly in the etiological features of influenza viruses. Therefore, further investigation with a larger sample is urgently needed to explore in-depth the etiological features of influenza virus infection.

Previous studies have suggested the possible coinfections of multiple viruses $[17,18]$. Ren et al. [18] demonstrated that viral pathogens were detected in $34.6 \%$ of ARIs samples, and $1.6 \%$ of the patients tested positive for more than one virus. Both double and triple infections were also found in this study, and the majority of $\mathrm{HBoV}$-positive cases were co-infected in a combined infectious state with other pathogens. These findings are consistent with those of a previous study [19]. Despite the lack of data on this topic, co-infections of influenza virus [20], HMPV [21] and/or seasonal coronaviruses (e.g., CoV-HKU1) [22] have been reported in adults and children infected with SARS-CoV-2, which can affect the morbidity and mortality of COVID-19. To the best of our knowledge, this study was the first to show that one COVID-19 case had concurrent infections with HPIV. These data on the co-infection of SARS-CoV-2 with common respiratory viruses or non-SARS-CoV-2 respiratory viral pathogens imply that the routine testing of multiple viral pathogens should not be ignored in mild epidemic areas, even during the COVID-19 outbreak. However, whether simultaneous viral infection in SARS-CoV-2 patients can potentially drive viral interference or impact disease outcome awaits further research.

In terms of laboratory features, most of the blood biochemical indicators were relatively similar between the patients with COVID-19 infection and those with other respiratory tract infections, except that the levels of TG and ALP were higher and lower respectively in COVID19 cases. These results suggest that SARS-CoV-2 infection exhibits similar features with many other respiratory virus infections. However, a recent study on the comparison of the clinical features between COVID-19 patients and non-SARS-CoV-2 virus-infected pneumonia cases demonstrated that COVID-19 patients had higher levels of aspartate aminotransferase, alanine aminotransferase, lactate dehydrogenase, $\gamma$-glutamyl transpeptidase and $\alpha$-hydroxybutyric dehydrogenase [23]. Thus, additional evidence is still required for the mechanism of higher TG but lower ALP levels in COVID-19 patients and the overall analysis of their clinical features.

\section{Conclusion}

This study analyzed the epidemiological patterns of different respiratory viruses, and found that although the widespread of COVID-19 is a serious public health concern, SARS-CoV-2 may not be the main pathogen responsible for respiratory tract infection in Southwest China, a mild epidemic area. Therefore, effective surveillance of the transmission patterns of respiratory tract pathogens in the COVID-19 affected area may be conducive for us to formulate an optimal treatment regimen and inhibit the rapid spread of the virus.

\section{Abbreviations}

COVID-19: Coronavirus disease 2019; SARS-CoV-2: Severe acute respiratory syndrome coronavirus 2; ARIs: Acute respiratory infections; 09H1N1: Influenza A (H1N1) 2009 virus; H3N2: Seasonal H3N2 virus; InfB: Influenza B virus; HADV: Human adenovirus; HBoV: Human bocavirus; HRV: Human rhinovirus; HPIV: Human parainfluenza viruses; HCOV: Human coronavirus (229E, OC43, NL63, HKU1); HRSV: Human respiratory syncytial virus; HMPV: Human metapneumovirus; Mp: Mycoplasma pneumonia; Ch: Chlamydia 


\section{Acknowledgements}

Not applicable.

\section{Authors' contributions}

YJS and ZZZ were responsible for lab experiments, statistical analysis, and manuscript preparation. RC was responsible for the revision of the manuscript. HYZ and TYHL were involved in lab experiments and statistical analysis. MJW and XBS were involved in evolving the ideas and revising the manuscript. WML and BWY were responsible for conception, study design, data interpretation and manuscript finalization. All authors have contributed to and have approved the final manuscript.

\section{Funding}

This study was supported by the Department of Science and Technology of Sichuan Province Program "2020YFS0004"; COVID-19 epidemic Science and Technology Project of West China Hospital of Sichuan University "HX2019nCoV066" and "HX2019nCoV008"; nCoV Emergency Project of Sichuan University "2020scunCoV Emergency 10003".

\section{Availability of data and materials}

The data and materials supporting the conclusions of the study are available from the corresponding author on reasonable request.

\section{Ethics approval and consent to participate}

Approval for this study was obtained from the Biomedical Research Ethics Committee of West China Hospital, Sichuan University (ChiCTR2000030542). Written informed consent was obtained from all participants before sample collection and the data analyzed in our study were anonymized before its use.

\section{Consent for publication}

Not applicable.

\section{Competing interests}

The authors declare that they have no competing interests.

\section{Author details}

'Department of Laboratory Medicine, West China Hospital, Sichuan University, 37 Guoxue Alley in the wuhou District, Chengdu, Sichuan, China. ${ }^{2}$ Department of Clinical Laboratory, The First People's Hospital of Shuangliu District, Chengdu/ West China (Airport) Hospital Sichuan University, Chengdu, Sichuan, China. ${ }^{3}$ Department of Respiratory and Critical Care Medicine, West China Hospital, Sichuan University, 37 Guoxue Alley in the wuhou District, Chengdu, Sichuan, China.

Received: 13 April 2020 Accepted: 2 September 2020

Published online: 21 September 2020

\section{References}

1. Novel Coronavirus (2019-nCoV) Situation Report-1, WHO. https://www.who. int/docs/default-source/coronaviruse/situation-reports/20200121-sitrep-1-201 9-ncov.pdf. Accessed 21 Jan 2020

2. Lai C-C, Shih T-P, Ko W-C, Tang H-J, Hsueh P-R. Severe acute respiratory syndrome coronavirus 2 (SARS-CoV-2) and coronavirus disease-2019 (COVID-19): the epidemic and the challenges. Int J Antimicrob Agents. 2020;55(3): 105924

3. Guan W-i, Ni Z-y, Hu Y, Liang W-h, Ou C-q, He J-x, Liu L, Shan H, Lei C-l, Hui DSC, et al. Clinical characteristics of coronavirus disease 2019 in China. N Engl J Med. 2020;382(18):1708-20.

4. Asner SA, Science ME, Tran D, Smieja M, Merglen A, Mertz D. Clinical disease severity of respiratory viral co-infection versus single viral infection: a systematic review and meta-analysis. PLoS One. 2014:9(6):e99392.

5. Finianos M, Issa R, Curran MD, Afif C, Rajab M, Irani J, Hakimeh N, Naous A, Haij M-J, Hajj P, et al. Etiology, seasonality, and clinical characterization of viral respiratory infections among hospitalized children in Beirut, Lebanon. J Med Virol. 2016:88(11):1874-81.

6. February 29, 2020, Update on the novel coronavirus pneumonia outbreak. http://www.nhc.gov.cn/xcs/yqtb/202003/9d462194284840ad96ce75eb8e4 c8039.shtml. Accessed 1 Mar 2020.
7. February 29,2020 , Update on the novel coronavirus pneumonia outbreak. http://wsjkw.sc.gov.cn/scwsjkw/gzbd01/2020/3/1/a83d26ef4e78484f9a2a3 bofb7cd91c0.shtml. Accessed 1 Mar 2020.

8. Li Y, Reeves RM, Wang X, Bassat Q, Brooks WA, Cohen C, Moore DP, Nunes M, Rath B, Campbell H, et al. Global patterns in monthly activity of influenza virus, respiratory syncytial virus, parainfluenza virus, and metapneumovirus: a systematic analysis. Lancet Glob Health. 2019;7(8):e1031-45.

9. Chen J, Hu P, Zhou T, Zheng T, Zhou L, Jiang C, Pei X. Epidemiology and clinical characteristics of acute respiratory tract infections among hospitalized infants and young children in Chengdu, West China, 20092014. BMC Pediatr. 2018;18(1):216.

10. Zhang $Y$, Yuan L, Zhang $Y$, Zhang $X$, Zheng M, Kyaw MH. Burden of respiratory syncytial virus infections in China: systematic review and metaanalysis. J Glob Health. 2015;5(2):020417.

11. Yu J, Xie Z, Zhang T, Lu Y, Fan H, Yang D, Bénet T, Vanhems P, Shen K, Huang F, et al. Comparison of the prevalence of respiratory viruses in patients with acute respiratory infections at different hospital settings in North China, 2012-2015. BMC Infect Dis. 2018;18(1):72.

12. Shi T, McLean K, Campbell H, Nair H. Aetiological role of common respiratory viruses in acute lower respiratory infections in children under five years: a systematic review and meta-analysis. J Glob Health. 2015;5(1): 010408.

13. Kong $D C$, Wu HY, Zheng YX, Pan H, Jiang CY, Zhang $X$, Chen J, Wu F. Etiologic and epidemiologic features of acute respiratory infections in adults from Shanghai, during 2015-2017. Zhonghua liu xing bing xue za zhi = Zhonghua liuxingbingxue zazhi. 2019:40(8):904-10.

14. Li J, Wang Z, Gonzalez R, Xiao Y, Zhou H, Zhang J, Paranhos-Baccala G, Vernet G, Jin Q, Wang J, et al. Prevalence of human metapneumovirus in adults with acute respiratory tract infection in Beijing, China. J Infect. 2012; 64(1):96-103.

15. Zhang L, Liu W, Liu D, Chen D, Tan W, Qiu S, Xu D, Li X, Liu T, Zhou R. Epidemiological and clinical features of human metapneumovirus in hospitalised paediatric patients with acute respiratory illness: a crosssectional study in southern China, from 2013 to 2016. BMJ Open. 2018;8(2): e019308.

16. Qi L, Xiong Y, Xiao B, Tang W, Ling H, Long J, Xiao D, Zhao H, Ye S, Chen S, et al. Epidemiological and Virological Characteristics of Influenza in Chongqing, China, 2011-2015. PLoS One. 2016;11(12):e0167866.

17. Li X, Li J, Meng L, Zhu W, Liu X, Yang M, Yu D, Niu L, Shen X. Viral etiologies and epidemiology of patients with acute respiratory infections based on sentinel hospitals in Gansu Province, Northwest China, 2011-2015. J Med Virol. 2018;90(5):828-35.

18. Ren L, Gonzalez R, Wang Z, Xiang Z, Wang Y, Zhou H, Li J, Xiao Y, Yang Q, Zhang J, et al. Prevalence of human respiratory viruses in adults with acute respiratory tract infections in Beijing, 2005-2007. Clin Microbiol Infect. 2009; 15(12):1146-53

19. Longtin J, Bastien M, Gilca R, Leblanc E, de Serres G, Bergeron MG, Boivin G. Human bocavirus infections in hospitalized children and adults. Emerg Infect Dis. 2008;14(2):217-21.

20. Wu X, Cai Y, Huang X, Yu X, Zhao L, Wang F, Li Q, Gu S, Xu T, Li Y, et al. Coinfection with SARS-CoV-2 and influenza a virus in patient with pneumonia, China. Emerg Infect Dis. 2020;26(6):1324-6.

21. Touzard-Romo F, Tapé C, Lonks JR. Co-infection with SARS-CoV-2 and Human Metapneumovirus. Rhode Island Med J (2013). 2020;103(2):75-6.

22. Lin $D$, Liu L, Zhang $M, H u$ Y, Yang Q, Guo J, Guo Y, Dai Y, Xu Y, Cai Y, et al, Co-infections of SARS-CoV-2 with multiple common respiratory pathogens in infected patients. Sci China Life Sci. 2020;63(4):606-9.

23. Zhao D, Yao F, Wang L, Zheng L, Gao Y, Ye J, Guo F, Zhao H, Gao R. A comparative study on the clinical features of coronavirus 2019 (COVID-19) pneumonia with other pneumonias. Clin Infect Dis. 2020;71(15):756-61.

\section{Publisher's Note}

Springer Nature remains neutral with regard to jurisdictional claims in published maps and institutional affiliations. 